Mechanics of

Materials and Structures

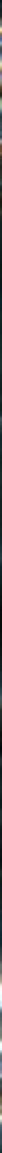

(a) 1 की

Volume 8 , No. 2-4

- April-June 2013

N. 


\title{
SELF-FOLDING OF A SLENDER MICROBEAM AND THIN FILM: AN ELASTICA MODEL
}

\author{
JiANLIN LIU AND JUNG HOON LEE
}

\begin{abstract}
Thin wires or films can be spontaneously folded into different shapes, and such phenomena hold promising applications in engineering, especially at micro and nanoscales. Based upon the established potential energy functional, we derived the governing equation and adhesive boundary condition for a self-folding system. Considering the inextensible condition of the structure, a closed-form solution for the deflection of a racket-like structure was obtained in terms of elliptical integrals, which applies for both macro and nanodimensions. We then determined the critical adhesive length under specified geometric and energetic constraints. The results show that self-folding is energetically favorable and thermodynamically stable with the cohesive work being strong enough and the structure being sufficiently flexible. As soon as the self-folding configuration is formed, the slender structure must possess an initial adhesive length. These conclusions are beneficial for the design of nanostructures, and the enhancement of their mechanical, chemical, optical, and electronic properties.
\end{abstract}

\section{Introduction}

Self-folding phenomena of thin films and wires, spanning the micro, macro, and nanoscales, are widely observable in nature and daily life. For example, when a piece of paper is coiled up in a tube, it does not make complete contact near its ends but makes a certain angle with the inner tube wall [Romero et al. 2008]. In addition, a flexible thin sheet can be self-folded or wrapped by capillary forces; Py et al. [2007; 2009] and Pineirua et al. [2010] carried out a series of experimental studies on such processes. They found that the surface tension of a droplet or a liquid film can cause the thin sheet to fold, origamilike, into different geometric shapes. They also provided numerical solutions to calculate the adhered configuration, which are consistent with their experimental results.

Nature often miraculously creates similar phenomena at different length scales; likewise, self-folding exists widely in the nanoworld. On the one hand, the creation of self-folded structures is technologically important in such practical applications as the self-assembly of nanostructures. A typical example is that silicon-germanium nanotubes can be formed by the self-folding of a thin film through an inexpensive label method [Schmidt and Eberl 2001]. During this process, the freed thin film is rolled up, with one of the film edges being brought into contact with the film itself and finally stuck by van der Waals forces [Glassmaker and Hui 2004]. Moreover, folding a two-dimensional graphene will significantly alter its properties, because the deformed morphology modifies the electronic band structure of the graphene and creates localized electronic states in the folded regions [Kim et al. 2011]. Using the folding method, one can control the shape of a graphene sheet, and then tailor its mechanical, chemical, optical, and electronic properties. On the other hand, self-folding or cross-section collapse can induce undesirable

Keywords: self-folding, elastica, adhesive boundary condition, energetic constraint, deflection. 
deformation or even failure of micro/nanosized devices and systems. For example, the circular cross section of a carbon nanotube (CNT) with a large diameter may collapse into a ribbon or dumbbell shape under van der Waals forces [Pantano et al. 2004]. Through molecular dynamic (MD) simulations, Gao et al. [1998] discovered that there are two possible configurations for a CNT in equilibrium state, one circular and one collapsed, corresponding to two determinate radius ranges, respectively. Recently, Tang et al. [2005] and Tang and Glassmaker [2010] studied the energetics of self-collapse of a single CNT by using the continuum mechanics method, and also calculated the critical radius for collapse.

The self-folding of thin films or microbeams may lead to different shapes, especially at micro and nanoscales [Cohen and Mahadevan 2003]. Like a microtubule inside a vesicle buckling into a racket-like shape [Fygenson et al. 1997], CNTs with a similar shape were also observed in a sample of high-pressure carbon monoxide single-walled nanotubes after 30 minutes of sonication in dichloroethane [Cohen and Mahadevan 2003]. The occurrence of self-folding in such small scaled materials as nanowires, microtubules, nanotubes, and thin films is mainly attributed to their high aspect ratios. More precisely, the maximal size (for example, the length of nanowire or the length/width of a thin film) is much larger than the persistence length [Zheng et al. 2004; Lee et al. 2007]. Due to carbon-carbon covalent bonding and seamless hexagonal network architecture, a CNT can be easily bent into an arc shape with significant curvature [Buehler et al. 2004]. This special racket-like configuration is actually an energetically favorable state, with the interplay of elastic deformation and van der Waals attraction between different parts of the CNTs.

Much effort has been directed toward understanding the physical mechanisms of self-folding processes. The first atomistic simulation of single-wall CNTs with very large aspect ratios subject to compressive loading was carried out in Buehler et al. [2004]. They investigated the shell-rod-wire transition of the mechanical behavior of CNTs with increasing aspect ratios. Following this work, Buehler et al. [2006] utilized atomistic simulation to study the deformation of a highly flexible nanotube forming a thermodynamically stable self-folded structure, and presented the critical length and critical temperature for folding or unfolding. Zhou et al. [2007] obtained the critical length for the self-folding of CNTs by MD simulations and infinitesimal deformation analysis. Mikata [2010] then derived an approximate solution for the self-folding of CNTs on the assumption that the curvature at the adhesion point is zero. Glassmaker and Hui [2004] modeled the CNT as an elastica, presented the close-formed differential equation set, and gave the numerical results. Similar to CNT folding, Cranford et al. [2009] studied the self-folding of mono and multilayer graphene sheets, utilizing a coarse-grained hierarchical multiscale model derived directly from atomistic simulation. Although the aforementioned studies have been devoted to selffolding problems, to date there is still a lack of systematic theoretical analysis on the underlying physical mechanisms, which involve very large deformation and strong geometric nonlinearity. In this paper, we present an elastica model to quantitatively study the mechanism of self-folding. Our motivation is to provide the explicit solution of the considered problem, then develop a new method to analyze the racket-like shape of self-folded structures via the classical elastica solution. This landmark theory, which can be traced back to the historic contribution of Euler [Love 1906], has been successfully used to solve some finite deformation problems of slender structures [Bisshopp and Drucker 1945; Liu and Feng 2007; Majidi 2007; Neukirch et al. 2012].

This article is organized as follows. In Section 2, we present a mechanics model to analyze the selffolding of structures in terms of energy, and derive the nonlinear governing equation and the adhesive 
boundary condition. In Section 3, we discuss the critical adhesive length under specified geometric and energetic constraints. Finally, we present the explicit solution for the deflection of a flexible structure.

\section{Elastica model}

2.1. Model formulation. First, we analyze the physical mechanisms of self-folding of a flexible structure, such as a filament or a thin film, by means of scaling laws. If the typical length of a planar structure is denoted as $L_{c}$, then the interfacial or surface energy $U_{S} \propto L_{c}$, the elastic strain energy $U_{E} \propto L_{c}^{2}$, and the potential energy due to gravity $U_{G} \propto L_{c}^{3}$ [Roman and Bico 2010]. As the dimension of a macroscopic fiber or film shrinks to nanometers, the effect of surface energy becomes significant and should be taken into account. Therefore, it can naturally be assumed that the effect of gravity is negligible. Given a sufficiently large flexibility or aspect ratio, the structure can reach an energetically stable self-folding shape like a tennis racket. For instance, a CNT can experience a shell-rod-wire transition as its aspect ratio increases [Buehler et al. 2004]. If a straight and slender CNT is subject to two compressive loads with magnitude of $P$ exerted at its two ends, it will buckle when the load is bigger than a critical value:

$$
P_{\mathrm{cr}}=\left(\frac{\pi}{L}\right)^{2} E I
$$

where $L$ is the total length of the CNT, $E I$ the bending stiffness of the CNT, $E$ the Young's modulus of the material, and $I$ the moment of inertia of the cross section of the beam. After it buckles, the CNT may become a ring-shaped structure [Bigoni 2012, 52-69] with radius $R$, and it can continue to be self-folded if the van der Waals force $q$ exceeds a critical value:

$$
q_{\mathrm{cr}}=\frac{3 E I}{R^{3}} .
$$

In this situation, the van der Waals force between the upper and lower portions of the CNT will attract each other, and the interfacial energy actually plays the role of a transmural pressure. In the present study, we only concentrate on the folded morphology of the glued CNT.

In what follows, we consider a slender filament or a sheet stuck by the adhesion force of the solid contact surface or a trapped liquid film, as shown in Figure 1a. Due to the symmetry and smoothness of this configuration, the upper half portion of the structure can be modeled as a beam with two clamped ends, as shown in Figure 1b. The total length of the $\operatorname{rod}$ is $L / 2$, and the adhered segment is $l$. We introduce a Cartesian coordinate system $(o-x y)$. Besides the Euler coordinates $x$ and $y$, the arc length $s$, a Lagrange coordinate, is also employed as a variable in our analysis. The slope angle of the beam at an arbitrary point is $\phi$, which continuously changes from $0^{\circ}$ at its left end to $-90^{\circ}$ at its right end.

According to Figure 1, the fixed boundary conditions of the elastic system are prescribed as

$$
\begin{gathered}
\phi(l)=0, \quad \phi\left(\frac{L}{2}\right)=-\frac{\pi}{2}, \quad \phi(L-l)=-\pi ; \\
y(l)=0, \quad y\left(\frac{L}{2}\right)=0 .
\end{gathered}
$$

The geometric conditions of the elastica are

$$
\dot{x}=\cos \phi, \quad \dot{y}=\sin \phi,
$$




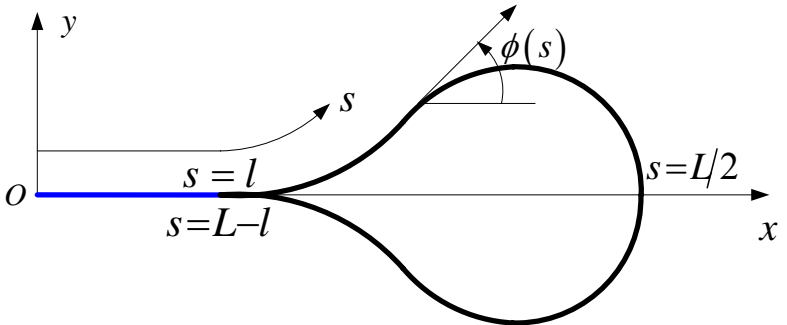

(a)

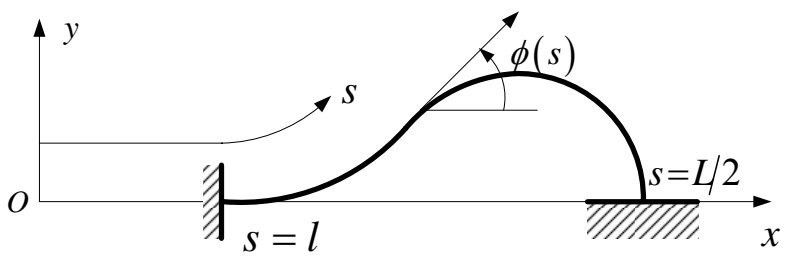

(b)

Figure 1. Schematic diagram of a slender structure self-folded into a racket-like shape. (a) The left segment is adhered by solid interface or liquid film. (b) The total length of the rod is $L$, the adhered segment $l$, and the slope angle at an arbitrary point $\phi$.

where a dot above a character stands for the derivative of a variable with respect to the arc length $s$.

The total potential energy of the system consists of two parts, namely the elastic strain energy and surface energy. Considering the symmetry of this configuration, the energy functional of the system can be written as

$$
\Pi=\int_{l}^{L / 2}\left[E I \dot{\phi}^{2}+\lambda_{1}(\dot{x}-\cos \phi)+\lambda_{2}(\dot{y}-\sin \phi)\right] d s-W_{a} l,
$$

where $\lambda_{1}$ and $\lambda_{2}$ are two Lagrange multipliers, enforcing the additional geometrical relations of (5).

The second term of at the right end of (6) stands for the surface energy, and the symbol $W_{a}$ is defined as the work of adhesion between two surfaces [Dupré 1969]. It is expressed as

$$
W_{a}=\left(\gamma_{1}+\gamma_{2}-\gamma_{12}\right) b
$$

where $b$ is the contact width out of the plane, $\gamma_{1}$ and $\gamma_{2}$ are the surface energies of the two different phases, and $\gamma_{12}$ the interfacial energy. In the conventional definition, the work of adhesion is actually the work per unit area necessary to create two new surfaces from a unit area of an adhered interface, which is a positive constant for any two homogeneous materials binding at an interface at a fixed temperature [Tang et al. 2005]. The cohesive work can be defined as the work of adhesion between two identical material interfaces, which reads

$$
W_{c}=2 \gamma_{1} b .
$$

For a slender structure with the interface adhered by a liquid film, the cohesive work or the interfacial energy per unit length is

$$
W_{c}=2\left(\gamma_{l}+\gamma_{\mathrm{SV}}-\gamma_{\mathrm{SL}}\right) b=2 \gamma_{l}\left(1+\cos \theta_{Y}\right) b
$$

where $\gamma_{\mathrm{SV}}, \gamma_{\mathrm{SL}}$, and $\gamma_{l}$ designate the interfacial tensions of the solid/vapor, solid/liquid, and liquid/vapor interfaces, respectively, and $\theta_{Y}$ is the Young's contact angle. At micro and nanoscales, the cohesive work is normally termed the binding energy $E_{B}$ [Zhou et al. 2007].

Although the intrinsic boundary condition of the present problem is fixed, we can view it as movable, and then the new variation scheme can be introduced (see Appendix A). During the variation operation, we should stress that the variation of the total potential energy can be separated to two portions: the first part $\delta \Pi_{1}$ is the variation when the adhesive point $s=l$ is assumed to be fixed; the second $\delta \Pi_{2}$ is the 
additional variation on the movable point at $s=l$. The additional term of the variation for the considered problem is because the adhesive length $l$ is yet to be determined, which leads to the movable boundary condition. Therefore, according to the principle of least potential energy one has

$$
\delta \Pi=\delta \Pi_{1}+\delta \Pi_{2}=0 .
$$

A detailed derivation of (10) can be found in Appendix B, and we can clearly obtain the governing equation of the elastica

$$
2 E I \ddot{\phi}-\lambda_{1} \sin \phi+\lambda_{2} \cos \phi=0
$$

and the adhesive boundary condition at the moving point

$$
E I \dot{\phi}(l)^{2}-W_{c}=0 .
$$

The symbols $\lambda_{1}$ and $\lambda_{2}$ in (11) can be easily identified as the horizontal and vertical components of the internal forces in the beam. It can be seen that the governing equation in (11) was obtained from the perspective energy functional, which conforms to the result derived by Tang and Glassmaker [2010], who adopted the analysis method of force equilibrium.

The transversality boundary condition represents the balance of the strain energy and the cohesive work, which is called the adhesive boundary condition or transversality condition in mathematical terminology. It is worth mentioning that the adhesive boundary condition in (12) can also be deduced via the concept of the $J$-integral in fracture mechanics, as described by Glassmaker and Hui [2004] in their analysis of silicon-germanium nanotube formation. The stationary potential and material force balance methods have also been adopted to derive the adhesive boundary condition [Majidi 2007]. The discrepancy between (12) and the result of [Glassmaker and Hui 2004] is due to a difference in geometry; the current model is symmetric in the configuration, in comparison with the single-side adhesive interface in the reference. In addition, the framework of the energy functional variation in consideration of the adhesive boundary condition can also be extended to solve other problems, where the boundary conditions can also be regarded as movable. The proposed method proves efficient and essential for calculating the contact angle of a droplet, the morphology of a cell, and the peeling of a CNT from the substrate [Seifert and Lipowsky 1990; Oyharcalbal and Frisch 2005; Bormasshenko and Whyman 2008].

Multiplying both sides of (11) by $\dot{\phi}$ and integrating, one has

$$
E I \dot{\phi}^{2}=D-\lambda_{1} \cos \phi-\lambda_{2} \sin \phi,
$$

where the parameter $D$ is an integration constant. Making use of (3), one obtains

$$
\begin{aligned}
E I \dot{\phi}(l)^{2} & =D-\lambda_{1}, \\
E I \dot{\phi}(L-l)^{2} & =D+\lambda_{1} .
\end{aligned}
$$

The symmetry of the configuration results in $\dot{\phi}(l)^{2}=\dot{\phi}(L-l)^{2}$, and then the Lagrange multiplier $\lambda_{1}=0$.

Considering (12) and (14), one has

$$
D=W_{c} .
$$


We introduce the parameter $\alpha=\sqrt{\lambda_{2} /(2 E I)}$, and replace $D /(2 E I)$ with $\alpha^{2} C$, where the symbol $C=$ $W_{c} / \lambda_{2}$. Apparently, the physical meaning of $\alpha$ is the square ratio between the internal force and the bending stiffness. Thus the governing equation (13) and the adhesive boundary condition (12) are respectively transformed into

$$
\frac{1}{2} \dot{\phi}^{2}=\alpha^{2}(C-\sin \phi), \quad \frac{1}{2} \dot{\phi}(l)^{2}=\alpha^{2} C .
$$

The combination of (14) and (17) 2 gives

$$
2 \alpha^{2} L_{\mathrm{ec}}^{2} C=1 .
$$

Here, we have defined a new characteristic length, that is, the elastocohesive length $L_{\mathrm{ec}}=\sqrt{E I / W_{c}}$, which is different from the elastocapillary length $L_{\mathrm{EC}}$ used in [Roman and Bico 2010]. The elastocohesive length can be equivalently written in another format, as the parameter $1 / \sqrt{Q}$ determined in [Glassmaker and Hui 2004]. For a slender structure adhered by a liquid film, the elastocohesive length $L_{\mathrm{ec}}=(\sqrt{2} / 2) L_{\mathrm{EC}}$ when $\theta_{Y}=0$. It can be seen that in this case, (14) and (18) are consistent with the result of [Py et al. 2007].

2.2. Displacement boundary condition. As $d s$ is always positive and the increment of the slope angle is not monotonic, (17) $)_{1}$ is simplified as

$$
\alpha d s=\frac{|d \phi|}{\sqrt{2(C-\sin \phi)}} .
$$

For convenient integration, the variable $\phi$ is replaced with $\theta$. They are related by

$$
(1+C) \sin ^{2} \theta=2 k^{2} \sin ^{2} \theta=1+\sin \phi \quad(0 \leq \theta \leq \pi, k>0),
$$

and

$$
C=2 k^{2}-1, \quad \sqrt{2(C-\sin \phi)}=2 k|\cos \theta|, \quad|d \phi|=\frac{2 k|\cos \theta|}{\sqrt{1-k^{2} \sin ^{2} \theta}} d \theta .
$$

In these equations, we consider the uniqueness of the solution in light of the physical meaning of the problem.

Substituting $(21)_{2}$ and $(21)_{3}$ into the displacement boundary condition in (3) leads to

$$
\alpha\left[y\left(\frac{L}{2}\right)-y(l)\right]=\int_{0}^{-\pi / 2} \frac{\sin \phi|d \phi|}{\sqrt{2(C-\sin \phi)}}=F(k, \pi)-F\left(k, \theta_{0}\right)-2\left[E(k, \pi)-E\left(k, \theta_{0}\right)\right]=0,
$$

where $\sin \theta_{0}=1 /(\sqrt{2} k)$ and $F(k, \theta)$ and $E(k, \theta)$ are the elliptic integrals of the first and second kinds, which are respectively defined as

$$
F(k, \theta)=\int_{0}^{\theta} \frac{1}{\sqrt{1-k^{2} \sin ^{2} \theta}} d \theta, \quad E(k, \theta)=\int_{0}^{\theta} \sqrt{1-k^{2} \sin ^{2} \theta} d \theta .
$$

2.3. Inextensible condition. To close this mathematical problem, we complement the inextensible condition of the elastic rod [Liu and Feng 2007; Neukirch et al. 2012], which is written as

$$
\alpha\left(\frac{L}{2}-l\right)=\int_{0}^{-\pi / 2} \frac{|d \phi|}{\sqrt{2(C-\sin \phi)}}=F(k, \pi)-F\left(k, \theta_{0}\right) .
$$




\section{Critical adhesion parameters}

3.1. Geometric constraint. From (22), one can solve for the corresponding values of $k=0.855092$ and $C=0.462366$, both of which are independent of the material parameters of the beam. Furthermore, (18) gives the following relation between the nondimensional internal force $\alpha L$ and the normalized elastocohesive length:

$$
\alpha L=\frac{1}{\sqrt{4 k^{2}-2}\left(L_{\mathrm{ec}} / L\right)},
$$

and the function curve is exhibited in Figure 2. It can be seen that the vertically internal force of the cross section on the beam will increase with the increase of the cohesive work. This means that the stronger interfacial adhesion ability will produce larger reaction force and internal force. The reduced vertical force $\alpha L$ can be solved from (25) provided that the elastocohesive length and the total length of the beam are given.

Substitution of (25) into (24) yields

$$
\frac{l}{L}=\frac{1}{2}-\sqrt{4 k^{2}-2}\left[F(k, \pi)-F\left(k, \theta_{0}\right)\right] \frac{L_{\mathrm{ec}}}{L} .
$$

The symbol $l$ in (26) is the critical adhesive length, which is a linear function of the elastocohesive length. This relation agrees with the previous scaling analysis [Py et al. 2007] and infinitesimal deformation calculations [Buehler et al. 2006; Zhou et al. 2007; Mikata 2010]. However, the slope of the linear function in (26) is about -3.03 and differs from the experimental result of -4.9 [Py et al. 2007]. In comparison, the previous work [Py et al. 2007] deals with the function of the attached length $(L-2 l)$ about the elastocapillary length, and the slope is approximately obtained by a linear regression method. The deviation between our theoretical result and their experiment may stem from their data processing method and the zero Young's contact angle assumption.

The linear relation in (26) also indicates that the adhesive length will be in the range of $0<l / L<0.5$, and the elastocohesive length meets the condition

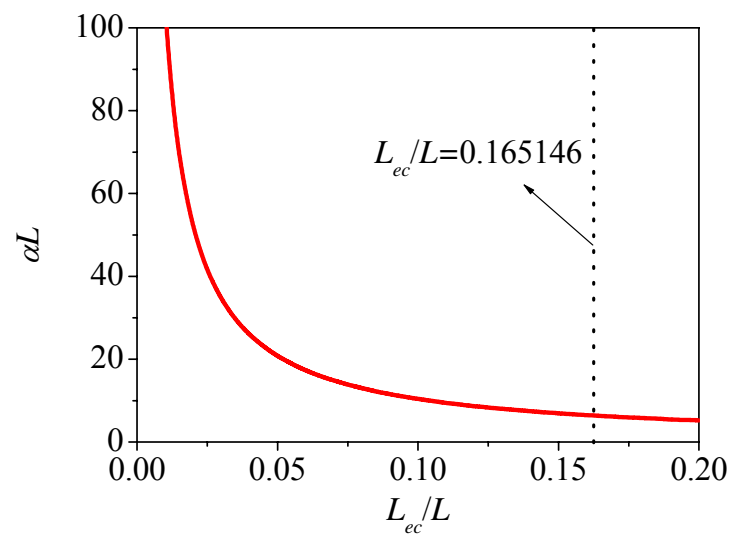

Figure 2. Variation of the nondimensional internal force with respect to the normalized elastocohesive length. 


$$
0<\frac{L_{\mathrm{ec}}}{L}<\frac{1}{2 \sqrt{4 k^{2}-2}\left[F(k, \pi)-F\left(k, \theta_{0}\right)\right]} .
$$

Equation (27) is an intuitively geometric constraint for the nondimensional cohesive length to ensure the self-folding shape. It indicates that this dimensionless length has an upper limit (as schematized in the dotted line in Figure 2, where $L_{\mathrm{ec}} / L=0.165146$ ), that is, the cohesive work must be strong enough, and simultaneously the rod should be sufficiently slender.

3.2. Energy constraint. Besides the aforementioned geometric constraint condition for the adhesive length, another stronger constraint can be derived from the consideration of energy. Using (17) , (19), $(21)_{2},(21)_{2}$, and (25), the analytical solution of the reduced potential energy on the system can be derived as

$$
\frac{\Pi L}{E I}=L \int_{l}^{L / 2} \dot{\phi}^{2} d s-\frac{L l}{L_{\mathrm{ec}}^{2}}=\frac{2 \sqrt{4 k^{2}-2}\left[F(k, \pi)-F\left(k, \theta_{0}\right)\right]}{L_{\mathrm{ec}} / L}-\frac{1}{2\left(L_{\mathrm{ec}} / L\right)^{2}} .
$$

The total potential energy is shown in Figure 3 as a function of the normalized elastocohesive length, which is not a monotonic function but has an extreme point. For a structure with extremely large elastocohesive length, its potential energy is higher than zero. In reality, the flexible structure takes a rectilinear shape for the ground state, whose total potential energy should be zero (there is no deformation). The thermodynamic law tells us that the self-folding is energetically favorable only if the current energy is smaller than that of the ground state. For a very short rod or a quite low cohesive work, the nondimensional elastocohesive length is very large, the total potential energy $\Pi$ can be higher than zero (see Figure 3), and it is difficult for the driving force to change the configuration from the ground state to the self-folding state. Therefore, there must exist a critical adhesive length to ensure a stable configuration. On the basis of (28), the energy requirement $\Pi<0$ leads to

$$
\frac{L_{\mathrm{ec}}}{L}<\frac{1}{4 \sqrt{4 k^{2}-2}\left[F(k, \pi)-F\left(k, \theta_{0}\right)\right]} .
$$

This energetic constraint condition gives a more strict limitation for the normalized elastocohesive length, which can only make sense in a much narrower range than that of (27). Beyond this scope, the

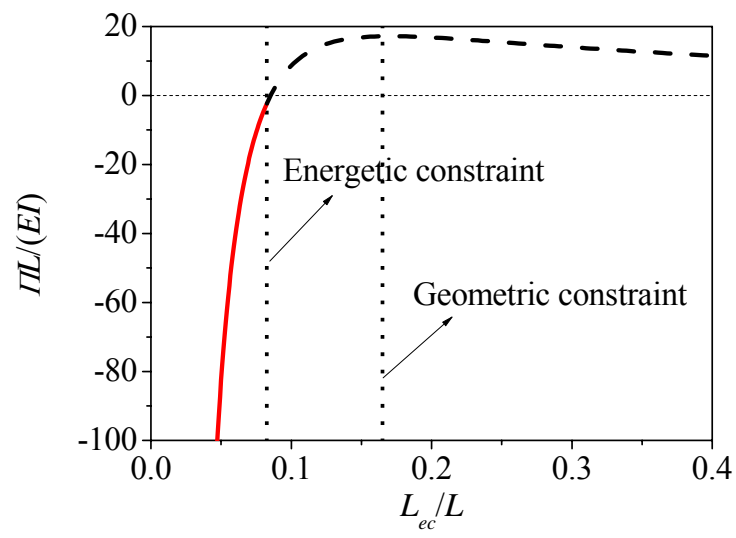

Figure 3. The dependence relationship between the nondimensional total potential energy of the system and the normalized elastocohesive length. 
racket-like shape of the structure is in a metastable state, as the dashed line shown in Figure 3. In other words, the elastocohesive length must meet both the energy constraint in (29) (the first vertical line in Figure 3) and the geometric constraint in (27) (the second vertical line in Figure 3), and the final adhesive length satisfies $1 / 4<l / L<1 / 2$. This newly presented conclusion indicates that, as soon as the selffolding configuration is formed, the slender structure must possess an initial adhesive length larger than $L / 4$. Otherwise, the racket-like morphology due to self-folding is not energetically favorable, and would seldom happen in reality. The corresponding critical total length to be self-folded is $L_{\mathrm{cr}}=12.11 L_{\mathrm{ec}}$ from (29), nearly equal to the calculated value $L_{\mathrm{cr}}=12.57 L_{\mathrm{ec}}$ in [Zhou et al. 2007].

3.3. Deflection. After the adhesive length has been determined, the deflection of the rod can be determined by

$$
\begin{aligned}
& \alpha x=\alpha \int_{0}^{s} \cos \phi d s=\alpha l+\int_{\theta_{0}}^{\theta} 2 k \sin \theta d \theta=\alpha l+\sqrt{4 k^{2}-2}-2 k \cos \theta, \\
& \alpha y=\alpha \int_{0}^{s} \sin \phi d s=F(k, \theta)-F\left(k, \theta_{0}\right)-2\left[E(k, \theta)-E\left(k, \theta_{0}\right)\right] .
\end{aligned}
$$

This is the analytical expression on the deflection of the elastica, which is different from the previous work, which was mostly conducted by numerical methods [Glassmaker and Hui 2004; Tang and Glassmaker 2010]. Equations (30) and (31) indicate that the nondimensional deflection of the rod is independent of the material parameters, showing the self-similarity of this racket configuration. The analytical solution of the displacement normalized by the total length of the beam reads

$$
\begin{aligned}
& \frac{x}{L}=\frac{1}{2}-\sqrt{4 k^{2}-2}\left[F(k, \pi)-F\left(k, \theta_{0}\right)\right] \frac{L_{\mathrm{ec}}}{L}+\frac{\sqrt{4 k^{2}-2} L_{\mathrm{ec}}}{L}\left(\sqrt{4 k^{2}-2}-2 k \cos \theta\right), \\
& \frac{y}{L}=\sqrt{4 k^{2}-2} \frac{L_{\mathrm{ec}}}{L}\left\{F(k, \theta)-F\left(k, \theta_{0}\right)-2\left[E(k, \theta)-E\left(k, \theta_{0}\right)\right]\right\},
\end{aligned}
$$

and the width of the loop can also be deduced as

$$
2 y_{\max }=\sqrt{4 k^{2}-2}\left\{F\left(k, \pi-\theta_{0}\right)-F\left(k, \theta_{0}\right)-2\left[E\left(k, \pi-\theta_{0}\right)-E\left(k, \theta_{0}\right)\right]\right\} L_{\mathrm{ec}} .
$$

The loop of the racket has the value $2 y_{\mathrm{max}}=1.236 L_{\mathrm{ec}}=0.874 L_{\mathrm{EC}}$, which is quite close to the numerical result of $0.89 L_{\mathrm{EC}}$ given in [Py et al. 2007].

Comparison with the experiments in [Py et al. 2007] shows that our exact solution successfully predicts the sheet wrapping behavior at the macro scale, and, in fact, it also applies to nanoscopic problems. For example, if the CNT is flexible and long enough, the gain in the potential energy due to van der Waals interaction (that is, the binding energy) is higher than the elastic strain energy necessary to bend the CNT. Under these circumferences, the tennis racket-like shaped CNTs can be formed. To simulate the morphology of self-folded CNTs, we select the following parameters [Mikata 2010]: Young's modulus $E=1000 \mathrm{GPa}$, moment of inertia $I=5.2678 \times 10^{-38} \mathrm{~m}^{4}$, binding energy $E_{B}=8.65 \times 10^{-11} \mathrm{~J} / \mathrm{m}$. Hence the elastocohesive length is $L_{\mathrm{ec}}=24.6778 \mathrm{~nm}$, and the corresponding critical length of the CNT is $L_{\mathrm{cr}}=298.861 \mathrm{~nm}$. We take the total length as $L=400 \mathrm{~nm}$ in computation. The deflection of the CNT self-folding is determined from (32) and plotted in Figure 4 under two representative values of the nondimensional elastocohesive length $L_{\mathrm{ec}} / L=0.0617$ (corresponding to $L_{\mathrm{ec}}=24.6778 \mathrm{~nm}$ ) and 0.05 , 


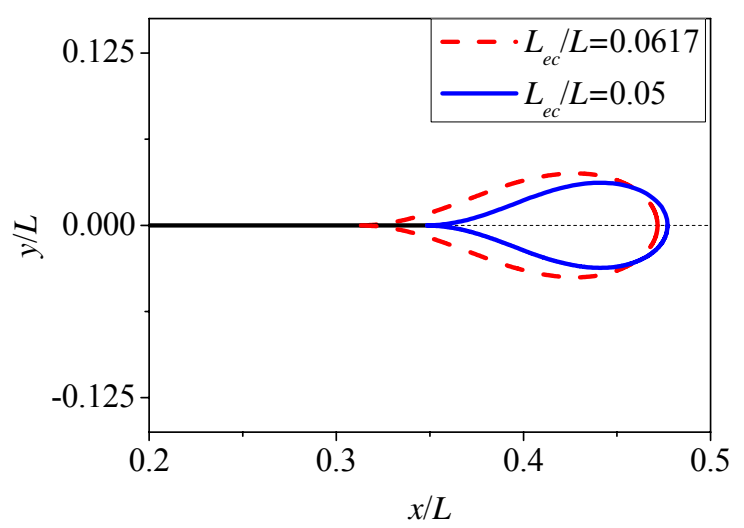

Figure 4. Normalized deflections of self-folding to a racket for a CNT, where $L_{\mathrm{ec}} / L=$ 0.0617 and $L_{\mathrm{ec}} / L=0.05$, respectively.

respectively. Clearly, the CNT will adhere more tightly with the increase of the cohesive work, and the width of the loop will shorten further.

\section{Conclusions}

We have presented an exact elastica model for analyzing the self-folding of slender structures. First, the potential energy functional of the system was constructed, which included the elastic strain energy and surface energy. We derived the governing equation and the adhesive boundary condition of the elastica, which stands for the balance of strain energy and surface energy at the movable boundary. Under the inextensible condition of the rod, we obtained the closed-form solution for the deflection of the beam in terms of elliptical integrals.

Furthermore, to ensure the self-folding of the slender structure into a tennis racket shape, the system must satisfy both the geometric and energetic constraints. The results show that self-folding is energetically favorable and thermodynamically stable with the cohesive work being strong enough and the structure being sufficiently flexible. The calculated result shows that, as soon as the self-folding configuration is formed, the structure must have an initial adhesive length after contact, of larger than $0.25 \mathrm{~L}$. The corresponding critical total length to be self-folded agrees well with the previous result.

This model can be used to investigate both a macroscopic sheet wrapped by a liquid film, and a CNT self-folded by van der Waals forces. In this paper, our attention is only on planar problems, while an extension of the presented elastica model to three-dimensional spatial problems, for example, the coiling of DNA [Manohar et al. 2008; Huang 2011], would be of great interest.

\section{Acknowledgements}

This project was supported by the National Natural Science Foundation of China (11272357 and 11320003), the opening project of the State Key Laboratory of Explosion Science and Technology (Beijing Institute of Technology) (KFJJ12-11M), and the Pioneer R\&D program for converging technology through the 
Korea Science and Engineering Foundation funded by the Ministry of Education, Science and Technology (Grant M10711270001-08M1127-00110). We also thank Prof. Xi-Qiao Feng for modifying the manuscript.

\section{Appendix A}

The transversality condition dealing with movable boundaries was in [Courant and Hilbert 1953, pp. 211214] and a more detailed deduction is presented here. A similar discussion can also be found in [Majidi et al. 2012].

Normally, the forced or fixed boundary conditions of an energy functional are prescribed as

$$
y(0)=y_{0}, \quad y^{\prime}(0)=y_{0}^{\prime} ; \quad y(a)=y_{a}, \quad y^{\prime}(a)=y_{a}^{\prime} .
$$

If the energy functional is expressed as

$$
\Pi[y(s, a)]=\int_{0}^{a} F\left[s, y(s, a), y^{\prime}(s, a), y^{\prime \prime}(s, a)\right] d s-\int_{a}^{L} W d s,
$$

then we consider the variation

$$
y(s)=y_{0}(s)+\epsilon Y(s),
$$

where $y_{0}(s)$ denotes the extreme solution to be found, and $s=a$ is a moving point to be determined.

According to the prescribed boundary condition of (A.1), the extreme and varied solution must fulfill

$$
y_{0}\left(a_{0}\right)=y_{a}, \quad y_{0}^{\prime}\left(a_{0}\right)=y_{a}^{\prime} ; \quad y(a)=y_{a}, \quad y^{\prime}(a)=y_{a}^{\prime} ; \quad Y(0)=Y^{\prime}(0)=0 .
$$

The unknown $a$ can be expanded as

$$
a(\epsilon)=a_{0}+\left.\epsilon \frac{d a}{d \epsilon}\right|_{\epsilon=0}+O\left(\epsilon^{2}\right) .
$$

The boundary value in (A.3) can also be expanded as

$$
\begin{aligned}
& y(a)=y_{0}\left(a_{0}\right)+\left.\epsilon y^{\prime}\left(a_{0}\right) \frac{d a}{d \epsilon}\right|_{\epsilon=0}+\epsilon Y\left(a_{0}\right)+O\left(\epsilon^{2}\right), \\
& y^{\prime}(a)=y_{0}^{\prime}\left(a_{0}\right)+\left.\epsilon y^{\prime \prime}\left(a_{0}\right) \frac{d a}{d \epsilon}\right|_{\epsilon=0}+\epsilon Y^{\prime}\left(a_{0}\right)+O\left(\epsilon^{2}\right) .
\end{aligned}
$$

Comparing (A.3), (A.6), and (A.7), one arrives at

$$
Y\left(a_{0}\right)=-\left.y^{\prime}\left(a_{0}\right) \frac{d a}{d \epsilon}\right|_{\epsilon=0}, \quad Y^{\prime}\left(a_{0}\right)=-\left.y^{\prime \prime}\left(a_{0}\right) \frac{d a}{d \epsilon}\right|_{\epsilon=0} .
$$

Equations (A.8) give the variation of the "new" end point $a(\epsilon)$ as a function of the variation in $y$ and the derivatives $y^{\prime}$ and $y^{\prime \prime}$ at the "old" end point $a_{0}$.

Before proceeding, we recall the formula for the derivative for an integral including a parameter $\alpha$. Let

$$
\phi(\alpha)=\int_{a(\alpha)}^{b(\alpha)} F(x, \alpha) d x .
$$


Its derivative with respect to $\alpha$ is

$$
\phi^{\prime}(\alpha)=\int_{a(\alpha)}^{b(\alpha)} F_{\alpha}(x, \alpha) d x+F[b(\alpha), \alpha] b^{\prime}(\alpha)-F[a(\alpha), \alpha] a^{\prime}(\alpha)
$$

Now let us return to the variation of the energy functional in (A.2). Substituting (A.1), (A.3), (A.4) and (A.8) into (A.2), and using (A.9), one can obtain the derivative of the functional

$$
\begin{aligned}
\left.\frac{d \Pi}{d \epsilon}\right|_{\epsilon=0}= & {\left.[W+F]_{s=a_{0}} \frac{d a}{d \epsilon}\right|_{\epsilon=0}+\int_{0}^{a_{0}}\left[F_{y} Y+F_{y^{\prime}} Y^{\prime}+F_{y^{\prime \prime}} Y^{\prime \prime}\right] d s } \\
= & {\left.[W+F]_{s=a_{0}} \frac{d a}{d \epsilon}\right|_{\epsilon=0}+\left[F_{y^{\prime}} Y+F_{y^{\prime \prime}} Y^{\prime}\right]_{0}^{a_{0}}+\int_{0}^{a_{0}}\left[F_{y} Y-\frac{d F_{y^{\prime}}}{d s} Y-\frac{d F_{y^{\prime \prime}}}{d s} Y^{\prime}\right] d s } \\
= & {\left.[W+F]_{s=a_{0}} \frac{d a}{d \epsilon}\right|_{\epsilon=0}+\left[F_{y^{\prime}} Y+F_{y^{\prime \prime}} Y^{\prime}-\frac{d F_{y^{\prime \prime}}}{d s} Y\right]_{0}^{a_{0}}+\int_{0}^{a_{0}}\left[F_{y}-\frac{d F_{y^{\prime}}}{d s}+\frac{d^{2} F_{y^{\prime \prime}}}{d s^{2}}\right] Y d s } \\
= & {\left.\left[W+F-y^{\prime} F_{y^{\prime}}-y^{\prime \prime} F_{y^{\prime \prime}}+y^{\prime} \frac{d F_{y^{\prime \prime}}}{d s}\right]_{s=a_{0}} \frac{d a}{d \epsilon}\right|_{\epsilon=0}+\left[F_{y^{\prime}} Y+F_{y^{\prime \prime}} Y^{\prime}-\frac{d F_{y^{\prime \prime}}}{d s} Y\right]_{0}^{a_{0}} } \\
& +\int_{0}^{a_{0}}\left[F_{y}-\frac{d F_{y^{\prime}}}{d s}+\frac{d^{2} F_{y^{\prime \prime}}}{d s^{2}}\right] Y d s=0 .
\end{aligned}
$$

The fact that the above integral equals zero leads to the Euler-Poisson equation

$$
F_{y}-\frac{d F_{y^{\prime}}}{d s}+\frac{d^{2} F_{y^{\prime \prime}}}{d s^{2}}=0
$$

The remainder of (A.11) comes to the following form

$$
\left.\left[W+F-y^{\prime} F_{y^{\prime}}-y^{\prime \prime} F_{y^{\prime \prime}}+y^{\prime} \frac{d F_{y^{\prime \prime}}}{d s}\right]_{s=a_{0}} \frac{d a}{d \epsilon}\right|_{\epsilon=0}=0
$$

This expression vanishes for arbitrary $d a /\left.d \epsilon\right|_{\epsilon=0}$, which corresponds to arbitrary $Y\left(a_{0}\right)$ if the bracket is zero. Then we can obtain the transversality condition for this variational problem:

$$
W=\left[y^{\prime} F_{y^{\prime}}+y^{\prime \prime} F_{y^{\prime \prime}}-y^{\prime} \frac{d F_{y^{\prime \prime}}}{d s}-F\right]_{s=a_{0}} .
$$

\section{Appendix B}

The detailed variation process on the energy functional proceeds as follows. Taking the variation on the energy functional, the principle of least potential energy leads to

$$
\delta \Pi=\delta \Pi_{1}+\delta \Pi_{2}=0 .
$$


The first part of the variation is under the assumption that the boundary condition is fixed, namely

$$
\begin{aligned}
& \delta \Pi_{1}=\int_{l}^{L / 2}\left[2 E I \dot{\phi} \delta \dot{\phi}+\delta \lambda_{1}(\dot{x}-\cos \phi)+\delta \lambda_{2}(\dot{y}-\sin \phi)\right.\left.+\lambda_{1} \delta \dot{x}+\lambda_{1} \sin \phi \delta \phi+\lambda_{2} \delta \dot{y}-\lambda_{2} \cos \phi \delta \phi\right] d s \\
&=\int_{l}^{L / 2}\left[-2 E I \ddot{\phi} \delta \phi+\delta \lambda_{1}(\dot{x}-\cos \phi)+\delta \lambda_{2}(\dot{y}-\sin \phi)\right. \\
&\left.\quad+\lambda_{1} \sin \phi \delta \phi-\lambda_{2} \cos \phi \delta \phi\right] d s+\left[2 E I \dot{\phi} \delta \phi+\lambda_{1} \delta x+\lambda_{2} \delta y\right]_{l}^{L / 2} .
\end{aligned}
$$

In view of the fixed boundary conditions (3) and (4), (B.2) can be reduced to

$$
\delta \Pi_{1}=\int_{l}^{L / 2}\left[\delta \lambda_{1}(\dot{x}-\cos \phi)+\delta \lambda_{2}(\dot{y}-\sin \phi)-\left(2 E I \ddot{\phi}-\lambda_{1} \sin \phi+\lambda_{2} \cos \phi\right) \delta \phi\right] d s-\lambda_{1} \delta l .
$$

Then we consider the corresponding variation on the movable boundary condition at the point $s=l$, which is written as

$$
\begin{aligned}
\delta \Pi_{2} & =-\left[E I \dot{\phi}^{2}+\lambda_{1}(\dot{x}-\cos \phi)+\lambda_{2}(\dot{y}-\sin \phi)-2 \dot{\phi}(E I \dot{\phi})-\dot{x} \lambda_{1}-\dot{y} \lambda_{2}\right]_{s=l} \delta l-W_{c} \delta l \\
& =\left[E I \dot{\phi}(l)^{2}-W_{c}+\lambda_{1}\right] \delta l .
\end{aligned}
$$

Considering the arbitrariness of the variation, one can obtain the following governing equation:

$$
2 E I \ddot{\phi}-\lambda_{1} \sin \phi+\lambda_{2} \cos \phi=0,
$$

and the transversality boundary condition at the moving point:

$$
E I \dot{\phi}(l)^{2}-W_{c}=0 .
$$

\section{References}

[Bigoni 2012] D. Bigoni, Nonlinear solid mechanics: bifurcation theory and material instability, Cambridge University Press, 2012.

[Bisshopp and Drucker 1945] K. E. Bisshopp and D. C. Drucker, "Large deflection of cantilever beams", Quart. Appl. Math. 3 (1945), 272-275.

[Bormasshenko and Whyman 2008] E. Bormasshenko and G. Whyman, "Variational approach to wetting problems: calculation of a shape of sessile liquid drop deposited on a solid substrate in external field", Chem. Phys. Lett. 463:1-3 (2008), 103-105.

[Buehler et al. 2004] M. J. Buehler, Y. Kong, and H. Gao, "Deformation mechanisms of very long single-wall carbon nanotubes subject to compressive loading", J. Eng. Mater. Technol. 126:3 (2004), 245-249.

[Buehler et al. 2006] M. J. Buehler, Y. Kong, H. Gao, and Y. Huang, "Self-folding and unfolding of carbon nanotubes", J. Eng. Mater. Technol. 128:1 (2006), 3-10.

[Cohen and Mahadevan 2003] A. E. Cohen and L. Mahadevan, "Kinks, rings, and rackets in filamentous structures", Pro. Natl. Acad. Sci. 100:21 (2003), 12141-12146.

[Courant and Hilbert 1953] R. Courant and D. Hilbert, Methods of mathematical physics, vol. 1, Interscience, New York, 1953. [Cranford et al. 2009] S. Cranford, D. Sen, and M. J. Buehler, "Meso-origami: folding multilayer graphene sheets", Appl. Phys. Lett. 95:12 (2009), 123121.

[Dupré 1969] A. Dupré, Théorie mécanique de la chaleur, Gauthier-Villars, Paris, France, 1969.

[Fygenson et al. 1997] D. K. Fygenson, J. F. Marko, and A. Libchaber, "Mechanics of microtubule-based membrane extension", Phys. Rev. Lett. 79 (1997), 4497-4500. 
[Gao et al. 1998] G. Gao, T. Çagin, and W. A. Goddard, III, "Energetics, structure, mechanical and vibrational properties of single-walled carbon nanotubes", Nanotechnology 9:3 (1998), 184-191.

[Glassmaker and Hui 2004] N. J. Glassmaker and C. Y. Hui, "Elastica solution for a nanotube formed by self-adhesion of a folded thin film”, J. Appl. Phys. 96:6 (2004), 3429-3435.

[Huang 2011] Z. Huang, "Modulating DNA configuration by interfacial traction: an elastic rod model to characterize DNA folding and unfolding", J. Biol. Phys. 37:1 (2011), 79-90.

[Kim et al. 2011] K. Kim, Z. Lee, B. D. Malone, K. T. Chan, B. Alemán, W. Regan, W. Gannett, M. F. Crommie, M. L. Cohen, and A. Zettl, "Multiply folded graphene", Phys. Rev. B 83:24 (June 2011), 245433.

[Lee et al. 2007] H. S. Lee, C. H. Yun, H. M. Kim, and C. J. Lee, "Persistence length of multiwalled carbon nanotubes with static bending", J. Phys. Chem. C 111:51 (2007), 18882-18887.

[Liu and Feng 2007] J.-L. Liu and X.-Q. Feng, "Capillary adhesion of microbeams: finite deformation analyses", Chin. Phys. Lett. $24: 8$ (2007), 2349-2352.

[Love 1906] A. E. H. Love, A treatise on the mathematical theory of elasticity, Second ed., Cambridge University Press, 1906.

[Majidi 2007] C. Majidi, "Remarks on formulating an adhesion problem using Euler's elastica (draft)", Mech. Res. Comm. 34:1 (2007), 85-90.

[Majidi et al. 2012] C. Majidi, O. M. O'Reilly, and J. A. Williams, "On the stability of a rod adhering to a rigid surface: shear-induced stable adhesion and the instability of peeling", J. Mech. Phys. Solids 60:5 (2012), 827-843.

[Manohar et al. 2008] S. Manohar, A. R. Mantz, K. E. Bancroft, C.-Y. Hui, A. Jagota, and D. V. Vezenov, "Peeling singlestranded DNA from graphite surface to determine oligonucleotide binding energy by force spectroscopy", Nano Lett. 8:12 (2008), 4365-4372.

[Mikata 2010] Y. Mikata, "Approximate solutions for a self-folding problem of carbon nanotubes”, J. Eng. Mater. Technol. 132:1 (2010), 011013.

[Neukirch et al. 2012] S. Neukirch, J. Frelat, A. Goriely, and C. Maurini, "Vibrations of post-buckled rods: the singular inextensible limit", J. Sound Vibr. 331 (2012), 704-720.

[Oyharcalbal and Frisch 2005] X. Oyharcalbal and T. Frisch, "Peeling off an elastica from a smooth attractive substrate", Phys. Rev. E 71 (2005), 036611.

[Pantano et al. 2004] A. Pantano, D. M. Parks, M. C. Boyce, and M. Buongiorno Nardelli, "Mixed finite element-tight-binding electromechanical analysis of carbon nanotubes”, J. Appl. Phys. 96 (2004), 6756-6760.

[Pineirua et al. 2010] M. Pineirua, J. Bico, and B. Roman, "Capillary origami controlled by an electric field", Soft Matter 6:18 (2010), 4491-4496.

[Py et al. 2007] C. Py, P. Reverdy, L. Doppler, J. Bico, B. Roman, and C. N. Baroud, "Capillary origami: spontaneous wrapping of a droplet with an elastic sheet”, Phys. Rev. Lett. 98:15 (Apr 2007), 156103.

[Py et al. 2009] C. Py, P. Reverdy, L. Doppler, J. Bico, B. Roman, and C. N. Baroud, "Capillarity induced folding of elastic sheets", Eur. Phys. J. Special Topics 166 (2009), 67-71.

[Roman and Bico 2010] B. Roman and J. Bico, "Elasto-capillarity: deforming an elastic structure with a liquid droplet", $J$. Phys.: Condensed Matter 22:49 (2010), 493101.

[Romero et al. 2008] V. Romero, T. A. Witten, and E. Cerda, "Multiple coiling of an elastic sheet in a tube", Proc. R. Soc. Lond. Ser. A Math. Phys. Eng. Sci. 464:2099 (2008), 2847-2866.

[Schmidt and Eberl 2001] O. G. Schmidt and K. Eberl, "Thin solid films roll up into nanotubes”, Nature 410:6825 (2001), 168.

[Seifert and Lipowsky 1990] U. Seifert and R. Lipowsky, "Adhesion of vesicles", Phys. Rev. A 42 (1990), 4768-4771.

[Tang and Glassmaker 2010] T. Tang and N. J. Glassmaker, "On the inextensible elastica model for the collapse of nanotubes", Math. Mech. Solid. 15:5 (2010), 591-606.

[Tang et al. 2005] T. Tang, A. Jagota, C.-Y. Hui, and N. J. Glassmaker, "Collapse of single-walled carbon nanotubes", J. Appl. Phys. 97 (2005), 074310.

[Zheng et al. 2004] L. X. Zheng, M. J. O’Connell, S. K. Doorn, X. Z. Liao, Y. H. Zhao, E. A. Akhadov, M. A. Hoffbauer, B. J. Roop, Q. X. Jia, R. C. Dye, D. E. Peterson, S. M. Huang, J. Liu, and Y. T. Zhu, "Ultralong single-wall carbon nanotubes", Nat. Mater. 3 (2004), 673-676. 
[Zhou et al. 2007] W. Zhou, Y. Huang, B. Liu, K. C. Hwang, J. M. Zuo, M. J. Buehler, and H. Gao, "Self-folding of single- and multiwall carbon nanotubes", Appl. Phys. Lett. $90: 7$ (2007), 073107.

Received 25 Sep 2012. Revised 11 Dec 2012. Accepted 26 Jan 2013.

JiANLIN LIU: liujianlin@upc.edu.cn

Department of Engineering Mechanics, China University of Petroleum, Qingdao, 266580, China

http://dem.upc.edu.cn/contents/38/96.html

JUNG HOON LEE: jleenano@snu.ac.kr

School of Mechanical and Aerospace Engineering, Seoul National University, Seoul 151-744, South Korea 


\title{
JOURNAL OF MECHANICS OF MATERIALS AND STRUCTURES
}

\author{
msp.org/jomms
}

\author{
Founded by Charles R. Steele and Marie-Louise Steele
}

EDITORIAL BOARD

ADAIR R. AGUIAR

KATIA BERTOLDI

University of São Paulo at São Carlos, Brazil

DAVIDE BIGONI

Harvard University, USA

IWONA JASIUK

University of Trento, Italy

Thomas J. PENCE

University of Illinois at Urbana-Champaign, USA

YASUHIDE SHINDO

Michigan State University, USA

DAVID STEIGMANN

Tohoku University, Japan

University of California at Berkeley

\section{ADVISORY BOARD}

J. P. CARTER University of Sydney, Australia

R. M. Christensen Stanford University, USA

G. M. L. GLADWELL University of Waterloo, Canada

D. H. Hodges Georgia Institute of Technology, USA

J. HUTCHINSON Harvard University, USA

C. HwU National Cheng Kung University, Taiwan

B. L. KARIHALOO University of Wales, UK

Y. Y. KIM Seoul National University, Republic of Korea

Z. Mroz Academy of Science, Poland

D. PAMPlonA Universidade Católica do Rio de Janeiro, Brazil

M. B. RubiN Technion, Haifa, Israel

A. N. SHUPIKOV Ukrainian Academy of Sciences, Ukraine

T. TARNAI University Budapest, Hungary

F. Y. M. WAN University of California, Irvine, USA

P. WRIGGERS Universität Hannover, Germany

W. YANG Tsinghua University, China

F. ZIEGLER Technische Universität Wien, Austria

PRODUCTION production@msp.org

SILVIO LEVY Scientific Editor

Cover photo: Mando Gomez, www.mandolux.com

See msp.org/jomms for submission guidelines.

JoMMS (ISSN 1559-3959) at Mathematical Sciences Publishers, 798 Evans Hall \#6840, c/o University of California, Berkeley, CA 94720-3840, is published in 10 issues a year. The subscription price for 2013 is US \$555/year for the electronic version, and $\$ 705 /$ year (+\$60, if shipping outside the US) for print and electronic. Subscriptions, requests for back issues, and changes of address should be sent to MSP.

JoMMS peer-review and production is managed by EditFLOW ${ }^{\circledR}$ from Mathematical Sciences Publishers.

PUBLISHED BY

mathematical sciences publishers

nonprofit scientific publishing

http://msp.org/

(C) 2013 Mathematical Sciences Publishers 


\section{Journal of Mechanics of Materials and Structures}

\section{Volume 8, No.:2-4 April-June 2013}

A diffuse cohesive energy approach to fracture and plasticity: the one-dimensional case

Gianpietro Del Piero, Giovanni Lancioni and Riccardo March 109

Continuum deployable shells made of thin plates

Vladimir A. GracheV and Yuriy S. NeUSTAdT

Self-folding of a slender microbeam and thin film: an elastica model

JIANLIN LIU and JUNG HoON LEE 169

Dispersion of guided waves in initially stressed layered plates

JIANGONG YU and SHENLEI LI 185

Empirical models for predicting protective properties of concrete shields against

high-speed impact

GABI BEN-Dor, ANATOLY DUBinsky and Tov ELPERIN

Transient response of a general anisotropic solid to dislocation growth: alternative formulation LOUIS MILTON BROCK 